\title{
DIAGNÓSTICOS DE ENFERMAGEM EM TRABALHADORES HIPERTENSOS DE UMA EMPRESA DE TRANSPORTE URBANO COLETIVO
}

\author{
NURSING DIAGNOSES FOUND WHITHIN INDIVIDUALS PRESENTING HYPERTENSION IN NA \\ URBAN TRANSPORTATION COMPANY

\section{DIAGNÓSTICOS DE ENFERMERÍA EN TRABAJADORES HIPERTENSOS DE UNA EMPRESA MUNICIPAL DE TRANSPORTE COLECTIVO URBANO}

\section{Rosa Maria Scanavini Pavan, Ivana Maria Passini Sodré Siviero², Vanessa Pellegrino Toledo ${ }^{3}$. Erika Cristiane Marocco Duran ${ }^{4}$}

RESUMO: O presente trabalho consistiu da análise, através de estudo exploratório, dos diagnósticos de enfermagem mais freqüentes em trabalhadores hipertensos de uma empresa municipal de transporte urbano na cidade de Araras/SP. O trabalho foi conduzido por graduandos e enfermeiras docentes de uma universidade do interior paulista, durante o ensino prático de sistematização da assistência de enfermagem. Foram atendidos 205 trabalhadores adultos efetivos da empresa e utilizado o histórico de enfermagem, sendo destacadas a entrevista de enfermagem e o exame físico direcionado para a hipertensão arterial, doença crônica que se caracteriza pelo aumento dos valores das pressões sistólica e/ou diastólica, afetando cerca de vinte milhões de brasileiros. Foram identificados os três diagnósticos (déficit de conhecimento sobre a doença; distúrbio do padrão de sono e nutrição alterada, com ingestão maior do que as necessidades corporais) por padrões de respostas humanas (conhecer, trocar, mover). A característica definidora levantada foi a verbalização do problema de falta de conhecimento sobre a doença - mesmo alguns trabalhadores já tendo recebido orientações médicas a respeito, e os fatores relacionados foram a falta de experiência prévia com esta patologia, baixa escolaridade e desinteresse quanto a maiores informações sobre a hipertensão arterial. Diante desses resultados, tornam-se prementes mais estudos direcionados à maior efetividade de propagação de informações sobre a doença e incentivos à detecção precoce, bem como aos procedimentos preventivos e à adesão aos tratamentos que possibilitarão a todos uma vida com qualidade e sem riscos.

PALAVRAS CHAVES: Diagnóstico de Enfermagem; Pressão Arterial; Cuidados de Enfermagem.

ABSTRACT: The present work sought an analysis of the most frequent nursing diagnoses found among individuals presenting abnormally high blood pressures - hypertension - in an urban transportation company in a city located in São Paulo state. The approaches used to evaluate such workers were: nursing interview and physical examination as to the hypertension, a disease affecting approximately 20 million brazilians, although there are not intense studies on this subject in brazil in our sample 15 men and 13 women presented these three most common diagnoses: lack of knowledge about the disease; disturbances on sleeping patterns; and altered nutrition, once there was ingestion of food higher than corporal needs. The lack of previous experiences related to hypertension, together with low schooling and little interest in knowing more about the disease represented the main causes impairing the individuals' adhesion to appropriate treatments and adoption of healthy daily habits. It is thus evident that it is necessary to take urgent steps towards people's conscientiousness about the importance of preventing and understanding the hypertension. It is only through studies and incentives aiming early detection and preventive measures that it will be possible to carry out effective nursing assistance to such a clientele.

KEYWORDS: Nursing Diagnosis; Blood Pressure; Nursing Care.

1 Mestranda em enfermagem, especialista em Saúde Familiar e docente pelo Centro Universitário Hermínio Ometto - UNIARARAS. E-mail:

rosamaria@uniararas.br , fone: 19-35671540.

2 Doutora em enfermagem pela Escola de Enfermagem de Ribeirão PretoUSP, orientadora do estudo.

3 Doutora em enfermagem pela Escola de Enfermagem de Ribeirão Preto USP, docente da Faculdade de Enfermagem do Centro Universitário Hermínio Ometto - UNIARARAS. E-mail: vanessatoledo@uniararas.br, Rua João Buzolin 250 casa 29, Jd. Das Flores - Araras - SP, CEP: 13607250.

,2

4 Doutoranda em enfermagem pela Escola de Enfermagem de Ribeirão Preto - USP, docente da Faculdade de Enfermagem do Centro Universitário Hermínio Ometto - UNIARARAS. 
RESUMEN: El presente trabajo consiste en un análisis, a través de estudio exploratorio, de los diagnósticos de enfermería más frecuentes en trabajadores hipertensos de una empresa municipal de transporte colectivo urbano en la ciudad de Araras, São Paulo. El trabajo fue conducido por los graduandos y por las enfermeras docentes de la facultad de enfermería, durante la enseñanza práctica de sistematización de la asistencia de enfermería. Fueron atendidos 205 trabajadores adultos efectivos de la empresa y fue utilizado el historico de enfermería, siendo destacadas la entrevista de enfermería y el examen físico direccionado para la hipertensión arterial, enfermedad crónica que se caracteriza por el aumento de los valores de las presiones sistólica y/o diastólica, afectando cerca de veinte millones de brasileños, a pesar de haber pocos estudios al respecto. Se identificaron los tres diagnósticos (déficit de conocimiento sobre la enfermedad, disturbio del patrón de sueño y nutrición alterada,

\section{INTRODUÇÃO}

A hipertensão arterial é uma doença crônica que se caracteriza pelo aumento dos valores das pressões sistólica e/ou diastólica, afetando cerca de 22,3\% a 43,9\% da população mundial adulta (MION \&TINUCCI, 1991).

No Brasil, embora inexistam estudos intensos, a estimativa é de que haja quase vinte milhões de indivíduos hipertensos. Há alta prevalência da hipertensão arterial na população adulta jovem, cerca de 10 a $20 \%$ nos países industrializados, sendo que $90 \%$ dos portadores sofrem de hipertensão arterial essencial ou primária e é desconhecida a causa básica da doença. Quando não tratada adequadamente, ocasiona graves conseqüências a órgãos vitais, como coração, cérebro e rins, que, uma vez comprometidos, podem implicar em seqüelas irreversíveis e levar à morte (ARAÚJO, 1999).

Diversos autores indicam procedimentos a serem empregados junto aos pacientes hipertensos, buscando seu envolvimento integral com o tratamento, e conseqüente controle da doença.

No que diz respeito ao diagnóstico da hipertensão, além dos níveis tencionais, os fatores de risco, a lesão de órgãos-alvo e as complicações associadas, qualquer classificação é insuficiente, há a necessidade de extrema cautela antes de rotular algum paciente como hipertenso, tanto pelo risco de falso-positivo, como pela repercussão em sua própria saúde além do custo social resultante (SANTELLO et al, 1996).

É importante que o enfermeiro, bem como todos os profissionais responsáveis pela assistência preventiva e curativa de saúde, esteja preparado para atender esta clientela específica, promovendo o autocuidado, no intuito de controlar a hipertensão e promover a melhora da qualidade de vida, que figura con una ingestión mayor a las necesidades corporales) por patrones de respuesta humana (conocer, cambiar, mover). La característica definidora levantada fue la verbalización del problema de falta de conocimiento sobre la enfermedad, a pesar de que algunos trabajadores ya habían recibido orientaciones médicas al respecto, y los factores relacionados fueron: falta de experiencia previa con esta patología, baja escolaridad y desinterés cuanto a mayores informaciones sobre la hipertensión arterial. Delante de estos resultados, se tornan necesarios más estudios direccionados a una mayor eficacia en la propagación de informaciones sobre la enfermedad e incentivos a la detección precoz, así como a los procedimientos preventivos y a la adhesión a los tratamientos que posibilitarán a todos una vida con calidad y sin riesgos.

PALABRAS CLAVES: Diagnóstico de Enfermería; Presión Arterial; Atención de Enfermería.

nos dias de hoje como um indicador competente dos resultados dos serviços de saúde (CIANCIARULLO, 1998).

Pensando nisso foram levantados os níveis tensionais dos trabalhadores de uma empresa de transporte urbano coletivo, com o propósito de avaliar os problemas de enfermagem mais freqüentes nesta população, utilizando um modelo de histórico de enfermagem por padrão de reação humana.

Ao planejar a assistência de enfermagem a ser estabelecida em cada caso, o enfermeiro lança mão do processo de enfermagem que lhe permite, de forma ordenada e científica, direcionar seus objetivos (GEORGE, 1993).

A teoria das necessidades humanas básicas surge como precursora deste modelo no Brasil, propondo etapas que direcionam a ação do enfermeiro, a saber: o histórico de enfermagem, o diagnóstico de enfermagem, um plano assistencial, um plano diário de cuidados, a evolução e o prognóstico (HORTA, 1971; HORTA, 1979).

Este modelo vem norteando o ensino da assistência de enfermagem desde a década de 70 , constituindo-se num referencial para os enfermeiros brasileiros, para tanto, são exigidos o raciocínio e a utilização permanente do saber, do fazer e dos dados do paciente, num contínuo fluir de informações, coordenadas pelo enfermeiro e transformadas em ações (CRUZ, 1993).

Apesar da forte influência deste modelo na enfermagem brasileira, há um consenso quanto à fase de diagnosticar ter sido deixada de lado na aplicação prática do processo de enfermagem, durante muitos anos. Ressalta-se a contribuição positiva para a autonomia do enfermeiro através dos diagnósticos de enfermagem, uma vez que referenciam as intervenções de enfermagem, exige um julgamento, 
favorecendo, assim, a exposição das ações do enfermeiro (CRUZ, 1995; GORDON, 1994).

O diagnóstico de enfermagem ou diagnóstico clínico feito por enfermeiros, descreve problemas de saúde reais ou potenciais, nos quais estes, em razão de sua formação e experiência, são capacitados e autorizados a dar tratamento (GORDON, 1994).

Ainda, pode ser abordado como um resultado, ou seja, a segunda etapa do processo de enfermagem, ou como um processo complexo de decisões que exige um corpo de conhecimentos específicos e habilidades cognitivas do enfermeiro (CARMAGNANI, 1999).

O esforço de enfermeiras americanas e canadenses, desde a década de setenta, na busca da unificação da terminologia utilizada pela enfermagem, resultou na criação, em 1982, da NORTH AMERICAN NURSING DIAGNOSIS ASSOCIATON - NANDA para sistematizar os avanços obtidos na área (NANDA, 2005).

Os diagnósticos de enfermagem propostos têm como base nove padrões de resposta humana: trocar, comunicar, relacionar, valorizar, escolher, mover, perceber, conhecer e sentir, constituindo a Taxonomia I. Atualmente já em nova versão, esta classificação foi inicialmente traduzida para nosso meio e resultou em fonte de muitos trabalhos para o aprimoramento dos diagnósticos de enfermagem na prática diária do enfermeiro, constando da taxonomia II da NANDA (NÓBREGA \& GARCIA, 1994; NANDA, 2005).

A terceira etapa do processo de enfermagem é o planejamento das intervenções de enfermagem e deve objetivar resultados concretos e mensuráveis ao longo da evolução do cliente.

Esta nova forma de atuação em si não garante a qualidade da assistência, se todo o processo não for controlado e avaliado constantemente, objetivando a identificação rápida de distorções e correções, de responsabilidade do enfermeiro na atenção ao trabalhador e à família (CIANCIARULLO, 1997).

Por outro lado, a aderência dos portadores de hipertensão arterial ao tratamento prescrito é uma preocupação do enfermeiro em contato com essa clientela, uma vez que, apesar da eficiência das medidas terapêuticas atualmente em voga, os níveis tensionais dos trabalhadores hipertensos freqüentemente não se encontram sob controle.

No que se refere à hipertensão arterial, observase que com a introdução do tratamento medicamentoso, surgem os efeitos colaterais indesejáveis e as complicações inerentes, em geral tardias, acarretando a não obtenção dos efeitos benéficos esperados pelo trabalhador.

Várias medidas têm sido propostas por parte dos enfermeiros para amenizar os conflitos que se impõem durante o tratamento medicamentoso, dentre elas a orientação sistematizada de enfermagem para o controle das doses, horários, indicações, efeitos colaterais, alimentação (composição, quantidade de calorias e de sal), hábitos de vida maléficos (fumo e bebida alcoólica), estresse, além do sedentarismo e do processo da doença propriamente dita.

Atuar junto aos trabalhadores hipertensos visando à promoção de condições favoráveis para o esclarecimento de dúvidas e adoção de hábitos e estilo de vida saudáveis, consta do caminho para o enfermeiro competente obter resultados positivos em relação ao autocuidado e aderência ao tratamento indicado.

Motivados por esse relevante aspecto de saúde pública que acomete silenciosamente grande parte da população, buscou-se aprofundar estudos no assunto, associando teoria e prática acadêmica, analisando segmentos da população trabalhadora que por questões de riscos da atividade podem estar mais vulneráveis ao problema.

\section{OBJETIVO}

- Analisar os diagnósticos de enfermagem mais freqüente em trabalhadores hipertensos, de uma empresa de transporte urbano coletivo.

\section{MÉTODOS}

Esta pesquisa constituiu-se de um estudo exploratório cuja população é de trabalhadores de uma empresa municipal de transporte urbano coletivo de uma cidade do interior paulista.

Durante o ensino prático de sistematização da assistência de enfermagem, foram atendidos 205 trabalhadores adultos efetivos de uma empresa de transporte urbano coletivo, pelos graduandos e enfermeiras docentes de uma universidade do interior paulista, no ano de 2004.

A coleta de dados foi feita durante a consulta de enfermagem através do histórico de enfermagem, e no momento do início da entrevista os participantes assinavam o termo de consentimento livre e esclarecido, no qual foi afirmado o sigilo e os preceitos éticos do estudo.

No instrumento utilizado para a consulta de enfermagem, destacaram-se duas partes:

1) Entrevista de enfermagem: A parte inicial continha dados de identificação, como nome, endereço, idade, sexo, escolaridade e profissão.

Incluiu também blocos de perguntas abertas e fechadas, orientadas pelos nove padrões de reações humanas que culminaram nos diagnósticos de enfermagem da taxonomia II da NANDA (2005).

2) Exame físico direcionado para a patologia hipertensão arterial: O exame físico foi efetuado com o trabalhador deitado na maca, após a realização da entrevista estando portanto descansado.

A verificação da pressão arterial era efetuada conforme a técnica do manguito (ATKINSON \& MURRAY, 1989).

As fichas com os dados sobre os trabalhadores foram então lidas e analisadas, sendo que após criteriosa observação, classificação e interpretação destes dados, identificaram-se os diagnósticos de 
enfermagem face aos problemas apresentados, registrando-os no impresso próprio, usando o referencial da Taxonomia II da NANDA (NANDA, 2005).

Foram consideradas na análise as variáveis de sexo, idade, estado civil, escolaridade, problemas de saúde relatados, salário, profissão, tipo de medicamento, obesidade, diabetes, alcoolismo, doenças cardíacas e acidente vascular encefálico como antecedentes familiares, fatores de risco do trabalhador, pressão arterial máxima, pressão arterial mínima, pulso, respiração, peso, altura, queixas verbalizadas pelo trabalhador, olhar, movimentos e expressão facial.

\section{RESULTADOS}

Os trabalhadores que constituíram a amostra deste estudo e que apresentaram hipertensão arterial foram 15 do sexo masculino e 13 do sexo feminino.

Neste grupo foram identificados os três diagnósticos de enfermagem por padrões de reações humanas, as características definidoras e os fatores relacionados, em relação à hipertensão arterial.

Os padrões que apareceram alterados foram 0 conhecer, o trocar e o mover.

Os três diagnósticos de enfermagem mais freqüentes foram déficit de conhecimento sobre a doença, distúrbio do padrão de sono e nutrição alterada com ingestão maior do que as necessidades corporais.

\section{Déficit de conhecimento sobre a doença}

Foi o diagnóstico mais freqüente encontrado.

Este diagnóstico refere-se ao padrão de resposta humana CONHECER e significa um estado no qual o trabalhador não tem a informação correta ou completa sobre aspectos necessários para manter ou melhorar seu bem estar (NÓBREGA \& GARCIA, 1994).

Este padrão envolve a compreensão adequada de uma informação e compreende as variáveis de conhecimento, aprendizagem e o processo de pensamento (MARIA, 1993).

A característica definidora que apareceu em todos os trabalhadores foi a falta de conhecimento sobre a doença, apesar de todos também terem informado que já tinham recebido orientações à respeito da doença pelo médico.

Pode-se inferir que esta orientação não tenha sido suficiente ou adequada. Talvez em razão de a maioria ser feita muito rapidamente durante a consulta e o longo período para retorno ao médico, cerca de um ou dois meses.

Nos fatores relacionados apareceram a falta de experiência prévia com esta doença em especial, dificultando o conhecimento e o déficit cognitivo.

Têm importância os outros problemas relacionados com a baixa escolaridade da maioria dos trabalhadores, que contribui para maior dificuldade em apreender sobre a doença e os cuidados; além do desinteresse identificado em alguns, pois mesmo que tenham a informação não pareceram interessados em aderir ao tratamento.

\section{Distúrbio do padrão do sono}

É o diagnóstico que pertence ao padrão de resposta humana MOVER e significa um estado no qual o trabalhador apresenta alteração no período do sono, causando desconforto ou interferindo no estilo de vida desejado (NÓBREGA \& GARCIA, 1994).

As características definidoras encontradas foram dificuldades para adormecer, sono interrompido e cansaço ao acordar.

Estes sinais podem, em parte, serem creditados a efeitos colaterais de medicamentos antihipertensivos ou a outras doenças associadas.

O trabalhador hipertenso costuma ficar irritado pela própria alteração da pressão que associada ao déficit de sono costuma fazer com que o paciente verbalize sensação de mal-estar.

A falta de sono prolongada traz, além do cansaço, a perda de concentração que resulta em dificuldade de manter o ritmo natural de vida.

Os fatores relacionados deste diagnóstico dizem respeito às alterações causadas pela própria doença e pela terapia medicamentosa, pois como já relatado, alguns agentes anti-hipertensivos podem provocar insônia.

\section{Diagnóstico de nutrição alterada com ingestão maior do que as necessidades corporais}

Está situado no padrão de resposta humana TROCAR e é definido como o estado no qual o trabalhador tem uma ingestão de nutrientes que excede as suas necessidades metabólicas (NÓBREGA \& GARCIA, 1994).

A característica definidora comum a todos os trabalhadores foi o peso 10 ou $20 \%$ acima do recomendado para a estatura e idade.

O fator relacionado identificado foi a não adesão à dieta prescrita mesmo com a presença de diabetes $\mathrm{e}$ colesterol elevado.

O excessivo uso de chás para o controle da hipertensão também parece contribuir para o descaso com a dieta, pois os trabalhadores acreditam que este hábito promova a isenção do controle alimentar.

\section{DISCUSSÃO}

O déficit de conhecimento sobre a hipertensão arterial ocasiona deficiências no autocuidado, favorecendo a exposição do trabalhador aos fatores de risco e complicações.

A literatura mostra que a obesidade pode contribuir para o aparecimento da hipertensão arterial e contribuir para outras complicações na saúde (OPARIL,1993). 
O trabalhador com excesso de peso, normalmente, tem uma vida mais sedentária e alimenta-se de maneira errada, formando um circulo vicioso e de rompimento difícil.

No que se refere ao distúrbio do padrão de sono, sabe-se que os ritmos biológicos são endógenos e não podem ser manipulados segundo a vontade do trabalhador.

As características de matutinidade e vespertinidade dos trabalhadores são importantes na determinação dos períodos de melhor desempenho e maior bem estar.

Estas informações podem ser utilizadas para otimizar a qualidade de vida, favorecendo os horários para a realização de atividades físicas.

A adesão de novos hábitos de vida é um processo de elaboração psíquica que exige vontade, empenho e disciplina, além da cooperação do grupo social do trabalhador sendo, portanto, um trabalho lento e complicado.

\section{CONSIDERAÇÕES FINAIS}

Por se tratar de um problema de saúde que, na maioria das vezes, ocorre de modo silencioso, mascarado pelas atribulações do cotidiano estressante do mundo atual, a hipertensão arterial pode conduzir a complicações secundárias importantes.

Torna-se, dessa forma de extrema urgência, que se promovam estudos sistemáticos e incentivos à detecção precoce, aos procedimentos preventivos, a adesão aos tratamentos, buscando conciliá-los da melhor maneira possível aos hábitos e estilo de vida do trabalhador, no interior das empresas.

Contribuindo para o fato, tem-se a importância da experiência profissional acadêmica, para que os alunos possam adensar suas percepções sobre o significado da doença e do autocuidado do trabalhador.

A sistematização da assistência de enfermagem provoca um cuidado individualizado $\mathrm{e}$, portanto, fidedigno perante as incapacidades proporcionadas pela doença.

As reflexões, suscitadas por este estudo, possibilitaram a elaboração de intervenções de enfermagem para os trabalhadores com hipertensão arterial, que se constituem num ponto de partida para novo estudo, contribuindo com o ensino, a pesquisa e a assistência de enfermagem.

Observa-se que somente com análise sistematizada e individualizada dos cuidados a serem aplicados ao trabalhador hipertenso é que se poderá obter, não só eficiência dos tratamentos (sejam preventivos ou curativos), como também eficácia nas respostas dos mesmos, propiciando um incremento da qualidade de vida em saúde e uma vida com qualidade.

\section{REFERÊNCIAS BIBLIOGRÁFICAS}

ARAÚJO, T. L. Hipertensão arterial - um problema de saúde coletiva e individual. Fortaleza: Fundação Cearense de Pesquisa e Cultura; 1999.

ATKINSON, L. D.; MURRAY, M. E. Fundamentos de Enfermagem: introdução ao processo de enfermagem. Rio de Janeiro: Guanabara - Koogan; 1989.

CARMAGNANI, M. I. S. Diagnósticos e Intervenções de Enfermagem em pacientes submetidos à laringectomia total e parcial. 1999. 165p. Tese (Doutorado) Escola de Enfermagem, Universidade de São Paulo, São Paulo.

CIANCIARULLO, T. I. Teoria e prática em auditoria de cuidados. São Paulo: Ícone Editora; 1997.

CIANCIARULLO, T. I. Cidadania e qualidade de vida. São Paulo: Ícone; 1998.

CRUZ, I. C. F. Diagnóstico de enfermagem: estratégia para sua formulação e validação. 1993. 154p. Tese (Doutorado) Escola de Enfermagem de Ribeirão Preto, Universidade de São Paulo, Ribeirão Preto.

CRUZ, D. A. L. M. A introdução do diagnóstico de enfermagem no ensino: sua influência no processamento de informações por alunos de graduação. 1995. 189p. Tese (Doutorado) Escola de Enfermagem, Universidade de São Paulo, São Paulo.

GEORGE, J.B. Teorias de enfermagem: a base para a prática da profissão. Porto Alegre: Artes Médicas; 1993.

GORDON, M. Nursing diagnosis, process and application. New York: Mosby; 1994.

HORTA, W. A. Observação sistematizada como base para o diagnóstico de enfermagem. Revista Brasileira de Enfermagem.n.24, p. 10-15, mar, 1971.

HORTA, W. A. Processo de enfermagem. São Paulo: EPU; 1979.

MARIA, V. L. R. Elaboração de diagnósticos de enfermagem do paciente coronariano em estado crítico. 1993. 208p. Tese (Doutorado) Escola de Enfermagem, Universidade de São Paulo, São Paulo.

MION, J.R.D.; TINUCCI, T. O grande desafio: como manter o paciente em tratamento com a pressão normalizada. São Paulo: DACHA; 1991.

NÓBREGA, N. M. L.; GARCIA, T. R. Uniformização da linguagem dos diagnósticos de enfermagem da NANDA. In: Sistematização das propostas do II Simpósio Nacional de Diagnósticos de Enfermagem; 1994; maio. 21-26; João Pessoa União, 1994.

NORTH AMERICAN NURSING DIAGNOSIS ASSOCIATION. Diagnósticos de Enfermagem da NANDA: definições e classificação - 2003 - 2004. North American Nursing Association; [trad.Cristina Correia]. Porto Alegre: Artmed; 2005. 300p. 
Diagnóstico de enfermagem em trabalhadores hipertensos de uma empresa de transporte urbano coletivo. Revista Eletrônica de Enfermagem, v. 07, n. 02, p. 173 - 178, 2005. Disponível em http://www.fen.ufg.br

ARTIGO ORIGINAL

OPARIL, S. Hipertensão arterial. In: Cecil, M. T. Tratado de Medicina Interna. Rio de Janeiro: Guanabara-Koogan, 1993. p. 345-58.

SANTELLO, J. L.; KRASILSIC, S.; MION, J. R. D. O papel da hipertensão arterial na prevenção primária e secundária das doenças cardiovasculares.
Revista da Sociedade Brasileira de Cardiologia. v. 6, n 5, p. 21-3, 1996.

Texto original recebido em 01/02/2005

Publicação aprovada em 30/08/2005 